\title{
Overexpression of small ubiquitin-related modifier-1 and sumoylated Mdm2 in oral squamous cell carcinoma: Possible involvement in tumor proliferation and prognosis
}

\author{
AKIHIRO KATAYAMA, TAKESHI OGINO, NOBUYUKI BANDOH, MIKI TAKAHARA, \\ KAN KISHIBE, SATOSHI NONAKA and YASUAKI HARABUCHI \\ Department of Otolaryngology-Head and Neck Surgery, Asahikawa Medical College, \\ Midorigaoka E 2-1-1-1, Asahikawa 078-8510, Japan
}

Received March 15, 2007; Accepted May 21, 2007

\begin{abstract}
The purpose of this research was to identify a molecular clue to tumor proliferation in oral squamous cell carcinoma (SCC) and to test the value as a predictive marker for prognosis. In cDNA array analysis, small ubiquitin-related modifier-1 (SUMO-1) was expressed at much higher levels in oral SCC tissue and oral SCC cell lines than normal oral epithelium. The result was confirmed by RT-PCR analysis and Western blot analysis. Transfection of the anti-SUMO-1 antisense oligonucleotide to oral SCC cells significantly reduced proliferation of the cells. Immunoprecipitation and Western blot analyses revealed that the oncoprotein Mdm2 was present predominantly as a form of SUMO-1 congestion (sumoylation) rather than as a non-sumoylated form in both oral SCC tissues and cell lines. Immunohistological analysis revealed that patients who showed coexpression of SUMO-1 and Mdm2 experienced more frequently local recurrence after initial treatments. Multivariate analysis confirmed that the dual-high expression of SUMO-1 and Mdm2 was an independent factor for local failure. These result suggested that overexpression of $\mathrm{Mdm} 2$ caused by overexpression of SUMO-1 may be involved in tumor aggressiveness even in patients with early stage oral SCC. SUMO-1 may be useful as a novel target for therapy in oral SCC as well as a clinical indicator for tumor recurrence together with Mdm2.
\end{abstract}

\section{Introduction}

A subset of patients with oral cavity squamous cell carcinoma (SCC), often of young age yet lacking a history of carcinogen exposure, has been identified with no clear etiology for tumor

Correspondence to: Professor Yasuaki Harabuchi, Department of Otolaryngology-Head and Neck Surgery, Asahikawa Medical College, Midorigaoka E 2-1-1-1, Asahikawa 078-8510, Japan

E-mail: hyasu@asahikawa-med.ac.jp

Key words: SUMO-1, Mdm2, local control, prognostic factor development. Genetic alterations have been recognized as important events in the carcinogenesis of oral SCC and have been used as predictors of progression risk.

Small ubiquitin-related modifier-1 (SUMO-1) is the best-characterized member of a rapidly growing family of ubiquitin-like proteins that are involved in post-translational modifications (1-3). SUMO-1 is reversibly attached to other proteins in an enzyme cascade that resembles ubiquitination. SUMO-1 congestion (sumoylation) affects the subcellular localization of substrates and stability as well as transcriptional activities. Over 20 proteins have been identified as sumoylation substrates, and many more sumoylation substrates are expected to exist. A relatively small number of oncoproteins, for example PML (4), c-Myb (5) and c-Jun (6), have been found to be substrates for sumoylation. The significance of sumoylation in the function of the oncogenes varies among them. Given the substrates involved, protein sumoylation would be expected to be important in the course of tumorigenesis and, accordingly, altered in human cancer (7). However, evidence to support this notion is still scarce.

$\mathrm{Mdm} 2$ is also a target protein for sumoylation $(8,9)$. $\mathrm{Mdm} 2$ is an ubiquitin ligase that promotes p53 ubiquitination and degradation (10-12). Mdm2 is also self-ubiquitinated and degraded. Lee et al (13) has shown that removal of SUMO-1 from sumoylated Mdm2 led to promotion of Mdm2 selfubiquitination, resulting in p53 stabilization. With regard to immunohistological studies using tumor tissue sections, many investigators have reported a relationship between Mdm2 and clinical characteristics of malignant tumors such as breast cancer $(14,15)$ esophageal carcinoma (16), hepatocellular carcinoma (17) and renal cell carcinoma (18). However, there is no previous information regarding clinical association of Mdm2 expression with head and neck SCC including oral SCC. Furthermore, association of SUMO-1 expression with clinical features is still unknown in any types of malignant tumor.

In a preliminary study, we performed cDNA array analysis to study the difference of gene expression profile among normal oral epithelium, oral SCC tissues and oral SCC cell lines. Among several candidate genes that expressed at higher levels in oral SCC, we focused on SUMO-1 as a novel gene for a molecular clue to tumor proliferation in oral SCC. In this study, using transfected oral SCC cells by 
anti-SUMO-1 antisense oligonucleotide, we showed that SUMO-1 plays a role in tumor proliferation. Using immunoprecipitation and Western blot analyses, we revealed that the Mdm2 was present predominantly as a sumoylated form rather than as a non-sumoylated form in oral SCC tissues and oral SCC cell lines. We performed immunohistological analysis of SUMO-1 and Mdm2 expressions in biopsy tissue samples from 57 patients with early stage oral SCC to test their value as predictive markers for prognosis.

\section{Materials and methods}

Cell culture. Three human oral squamous cell carcinoma (SCC) cell lines, SAS, HSC3 and HSC4 were used in this study. They were all obtained from the Health Science Research Resources Bank (Osaka, Japan). The cell lines were established from oral SCC. The cells were cultured in RPMI-1640 (Gibco, Auckland, NZ) medium supplemented with $10 \%$ fetal bovine serum (FBS), $50 \mathrm{U} / \mathrm{ml}$ penicillin, and $50 \mu \mathrm{g} / \mathrm{ml}$ streptomycin (Life Technologies, Inc., Gaithersburg, MD) at $37^{\circ} \mathrm{C}$ with $5 \%$ $\mathrm{CO}_{2}$.

Patients and tissue samples. For cDNA array analysis, RT-PCR analysis and Western blot analysis, oral SCC tissues and normal oral epithelium were resected from 5 patients before treatment and stored at $-80^{\circ} \mathrm{C}$ until use. Tissue samples of oral SCC and normal oral epithelium were confirmed microscopically at hematoxylin-eosin stained sections.

For immunohistological analysis, the study group consisted of 57 Japanese patients (44 males and 13 females) who were diagnosed with oral SCC in early stages at Department of Otolaryngology-Head and Neck Surgery, Asahikawa Medical College between 1976 and 2003. All patients signed informed consent for therapy and tissue studies that had received prior approval by the Institutional Review Board. Clinical features and initial therapies of the patients are listed in Table I. Follow-up period ranged from 5 to 222 months with a median of 54 months.

cDNA array analysis. Human cDNA expression array membranes were purchased from Clontech (Atlas Human Cancer 1.2 array; Mountain View, CA). The mRNA samples were reverse transcribed into cDNA using a mixture of array gene-specific primers and deoxynucleotide triphosphate (dNTP) including [ $\left.{ }^{32} \mathrm{P}\right]-\mathrm{dATP}$. The cDNA was purified using NucleoSpin Extraction Spin columns (Clontech, Mountain View, CA) hybridized to array membranes according to manufacturer's manual, exposed to a phosphoimaging screen at room temperature for $30 \mathrm{~min}$, and then scanned using BAS2000 (Fuji Photo Film, Tokyo, Japan). A gild was applied to the images of hybridization spots, followed by quantification of spot intensity using BAStation version 1.31 (Fuji Photo Film) software. Background signals were defined as the average of the hybridization signals produced by 9 negative controls on the array. All hybridization signals were normalized to those of action and glyceraldehydes 3-phosphate dehydrogenase.

$R T-P C R$. Total cellular RNA from oral SCC cell lines, oral SCC tissues and normal oral epithelium was extracted with
Table I. Clinical and histologic features in 57 patients with early stage oral squamous cell carcinoma.

Characteristic

No. of cases $(\%)$

Gender

Female

Male

Age (years): median 54, range 42-84

$<60$

$\geq 60$

Primary sites

Tongue

Floor of mouth

Clinical stage

I (T1N0M0)

II (T2NOMO)

Tumor differentiation

Well

Moderately

Poorly

Initial therapy

Surgery alone

Radiotherapy alone

Combined with surgery and radiotherapy

$27(47)$

As an initial therapy, 5 patients were treated with local irradiation alone with total dose of $50 \mathrm{~Gy} ; 2$ patients were with external beam and 3 were combined with internal implant of Cs- 137 and external beam. Twenty-five patients were treated with surgical resection with laser scalpel. The remaining 27 patients were treated with surgery following preoperative irradiation with external beam $40 \mathrm{~Gy}$.

SV Total RNA Isolation system (Promega Corp., Madison, WI). The first strand cDNA was synthesized using MMLV reverse transcriptase (Gene Hunter, Inc., Nashville, TN) with Oligo dT-primers (Applied Biosystems, Forster City, CA) for $60 \mathrm{~min} 37^{\circ} \mathrm{C}$ according to manufauturer's instructions. Oligomer primers were synthesized for SUMO-1 (sense: 5'gtctgaccaggaggcaaaaccttcaa-3'; antisense: 5'-aactgttgaatga cccccgtttgtt-3'), $\beta$-actin (sense: 5'-atgggtcagaaggattcctatgt-3'; antisense: 5'-tcaggaggagcaatgatcttga-3') (Sigma Genosis, Tokyo, Japan). PCR was performed in a $10 \mu 1$ reaction mixture containing $4.95 \mu \mathrm{l} \mathrm{H} \mathrm{H}_{2} \mathrm{O} ; \mu \mathrm{l} \mathrm{PCR}$ buffer (contains $15 \mathrm{mM} \mathrm{MgCl} 2$, 10-fold concentrated); $1 \mu 1$ deoxynucleoside triphosphate (dNTPs, $2 \mathrm{mM}) ; 1 \mu 1$ forward primer $(2 \mathrm{mM})$; $1 \mu 1$ reverse primer $2(2 \mathrm{mM}) ; 0.05 \mu 1$ Taq DNA polymerase (AmpliTaq Gold; Applied Biosystems, Forster City, CA, 5 U/ $\mu 1) ; 1 \mu 1 \mathrm{cDNA}(100 \mathrm{ng} / \mu \mathrm{l})$. Thirty cycles of denaturation $\left(94^{\circ} \mathrm{C}, 1 \mathrm{~min}\right)$, annealing $\left(58^{\circ} \mathrm{C}, 1 \mathrm{~min}\right)$ and extension $\left(72^{\circ} \mathrm{C}\right.$, 1 min) were carried out in a DNA thermal cycler (Programmable Thermal Controller PTC-100; MJ Research, Inc., Waltham, MA). 
Oligonucleotide and transfection. High-performance liquid chromatography purified anti-SUMO-1 antisense oligonucleotide and control oligonucleotide, both of them with phosphorotioate modification and FITC-labeling, were purchased from Sigma Genosis. The cDNA sequence of SUMO-1 was obtained from GenBank. As anti-SUMO-1 antisense oligonucleotide, a 19-mer oligonucleotide complementary to the translation start codon region of SUMO-1 was prepared (5'-tggtcagacatgatgacgg-3') (Sigma Genosis). A second 19-mer oligonucleotide was designed to contain the same number and types of base as the antisense oligonucleotide, but in a random order (5'-accagtctgtact actgcc-3') as control oligonucleotide. The random sequence was determined as non-complementary to any sequence in GenBank. Both the antisense and random oligos were prepared with phosphrothioate modification and stored at $-20^{\circ} \mathrm{C}$. Transfection of oligonucleotide was performed using Lipofectin (Life Technologies) as carrier. Prior to transfection, SAS were planted at a density of $10^{5}$ cells/well in 6-well plates (Costar, Cambridge, MA) and grown for $24 \mathrm{~h}$. The transfection mixture was prepared in RPMI-1640 containing $1.5 \mu \mathrm{l}$ Lipofectin per $100 \mathrm{nM}$ oligonucleotide according to the manufacturer's instruction. Final concentrations of oligonucleotide were $0,10,50,100$ and $200 \mathrm{nM}$. Transfection was carried out for $4 \mathrm{~h}$ in a total volume of $1 \mathrm{ml}$ per well followed by removal of transfection mixture and addition of complete culture medium.

MTT assay. SAS cells were planted $2 \times 10^{3}$ cells/well in 96-well plate for the 12,24 , and $36 \mathrm{~h}$ in $100 \mu \mathrm{l}$ RPMI-1640 with $0.5 \%$ BSA $100 \mu 1$ after transfection of oligonucleotides at $100 \mathrm{nM}$ concentrations. To determine the number of viable cells, we used the Cell Titer One solution Cell Proliferation Assay (Promega Corp., Madison, WI). Twenty $\mu 1$ methyltiazoletetrazolium (MTT) solution was added to each well, and after 2-h incubation at $3^{\circ} \mathrm{C}$, the absorbance at $490 \mathrm{~nm}$ was measured by ELISA plate reader (NJ-2300; Nalge Nunc International, Tokyo, Japan). The experiments were performed 5 times.

Immunoprecipitation and Western blot analyses. Whole lysates from oral SCC cell lines, oral SCC tissues and normal oral epitheliums were prepared by adding $200 \mu 1$ ice-cold lysis buffer $[0.5 \%$ Triton X-100, $50 \mathrm{mM}$ Tris ( $\mathrm{pH} 7.2), 140 \mathrm{mM}$ $\mathrm{NaCl}, 10 \mathrm{mM}$ EDTA, $50 \mathrm{mM} \mathrm{NaF}$ and $1 \mathrm{mM} \mathrm{Na}_{3} \mathrm{VO}_{4}$ ) containing the protease inhibitor cocktail Complete Mini (Roche, Mannheim, Germany]. Lysates were clarified by centrifugation and immunoprecipitated with Ab bound to Protein A/G Sepharose Fast Flow (Amersham Pharmacia Boitech, Uppsala, Sweden) for $90 \mathrm{~min}$ at $4^{\circ} \mathrm{C}$. The resulting immunocomplexes were washed and run on $4-12 \%$ Bis-Tris SDS-PAGE gels (Invitrogen, San Diego, CA). Proteins were then blotted onto Immobilon-P (Millipore, Belford, MA), blocked in 5\% skim milk, and probed with mouse anti-human SUMO-1 monoclonal antibody (Zymed, South San Francisco, CA) and mouse anti-human Mdm2 monoclonal antibody (Oncogene, Boston, MA) followed by goat anti-mouse IgG-HRP (Amersham Phermacia Biotech, Backinghamshire, England). ECL system (Amersham Phermacia Biotech) was used for detection.
Immunohistological staining. Formalin-fixed and paraffinembedded specimens were obtained from surgical biopsies at the pretreatment period. The specimens were cut in $5-\mu \mathrm{m}$ sections. The slides were deparaffinized in xylane and ethanol. These sections were then incubated with $3 \%$ hydrogen peroxide for $30 \mathrm{~min}$. The slides were placed in $10 \mathrm{mM}$ citric acid buffer containing $0.1 \%$ Tween-20 at $\mathrm{pH} 6.0$ and underwent antigen retrieval for $20 \mathrm{~min}$ at $750 \mathrm{~W}$ and $95^{\circ} \mathrm{C}$ in a microwave oven. Mouse anti-human SUMO-1 monoclonal antibody (Oncogene) at 1:1000 dilution and mouse anti-human Mdm2 monoclonal antibody (Oncogene, $2.5 \mu \mathrm{g} / \mathrm{ml}$ ) were used. These primary antibodies were incubated overnight at $4{ }^{\circ} \mathrm{C}$, followed by incubation with peroxidase-labeled dextran polymer, EnVision $^{+}$(Dako A/S, Carpinteria, CA) for $30 \mathrm{~min}$ at room temperature (19). They were visualized by immersing the slides in freshly prepared $0.02 \%$ diaminobenzidine solution for $10 \mathrm{~min}$. The sections were finally counterstained with Lillie-Mayer's hematoxylin and mounted. The sections were examined microscopically by three of the authors (A. Katayama, T. Ogino and N. Bandoh) without knowledge of clinico-pathological features under magnification x200. More than 1000 tumor cells were counted to calculate the staining ratio of tumor cells. The positive staining with SUMO-1 was defined when cytoplasm and/or nuclear staining was observed. The positive staining with Mdm2 was defined when nucleic staining.

Statistical analysis. Correlation between two variables was tested by Spearman rank correlation coefficient and was summarized with the appropriate p-value. Two groups were compared using Mann-Whitney U test, and were summarized with their appropriate p-value. Time was defined as the period from diagnosis to the target event or last follow-up. For overall survival (OS), the target event was death. For cause-specific survival (CSS), it was cause-specific death. For local control (LC), it was local failure. For regional and distant control (RDC), it was regional lymph node and/or distant metastases. The probability of OS, CSS, LC and RDC were calculated using Kaplan-Meier method and compared using log-rank test. For determination of factors related to OS, CSS, LC and RDC, a Cox proportional hazards model was used. The final results of these analyses are hazard ratios (HR), their 95\% confidence intervals (CI) and p-value. The p-values less than 0.05 were considered to be statistically significant.

\section{Results}

Overexpression of SUMO-1 gene in oral SCC. In a preliminary study, we performed cDNA array analysis to survey the difference of gene expression profiles among normal oral epithelium, oral SCC tissues and oral SCC cell lines. Of 1176 genes screened on the array membrane, ten genes expressed at higher levels in oral SCC. SUMO-1 was one of the genes that expressed in oral SCC at much higher levels than in normal oral epithelium. As shown in Fig. 1A, the expression levels of SUMO-1 in SAS cells, HSC-3 cells, and HSC-4 cells that were from oral SCC and oral SCC tissue were, respectively, 7.83, 7.23, 11.91 and 6.50 times higher than that in normal oral epithelium (Fig. 1A). 


\section{A

Normal oral
epithelium

SCC cell lines

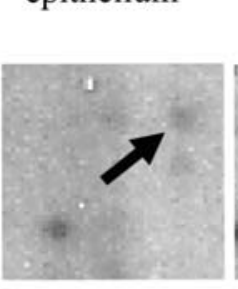

ratio

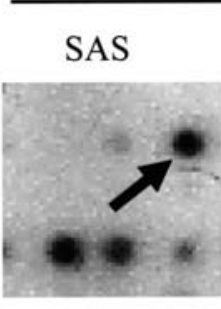

$\mathrm{X} 7.83$
HSC3

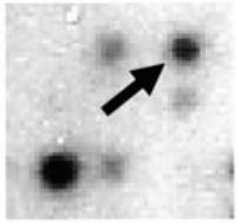

$\mathrm{X} 7.23$

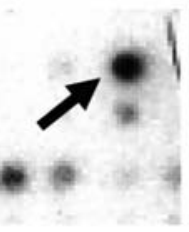

$\mathrm{X} 11.91$

\section{SCC tissue}

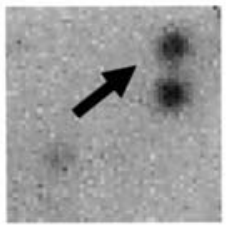

$\mathrm{X} 6.50$

B

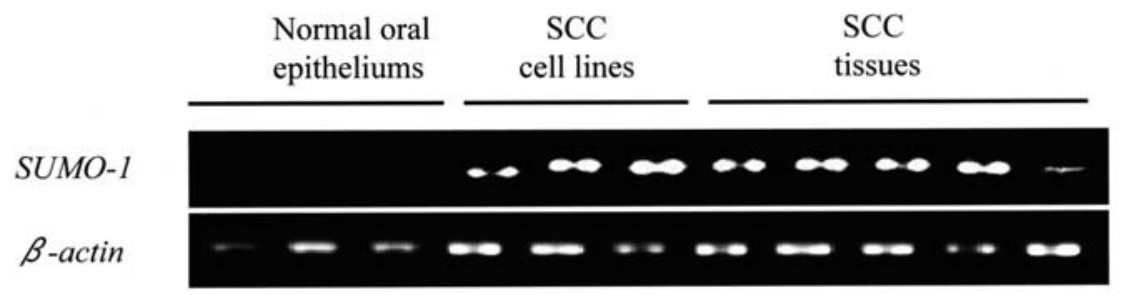

Figure 1. Expression of SUMO-1 by oral SCC. (A) cDNA array analysis. SUMO-1 (black arrows) is present in 3 oral SCC cell lines and a SCC tissue at 7.83, 7.23, 11.91 and 6.50 times normal oral epithelium level, respectively. (B) RT-PCR analysis of SUMO-1 gene expression. SUMO-1 gene expressions were detectable in all oral SCC cell lines and tissues, but almost negative in normal oral epitheliums.

In RT-PCR analysis, the $S U M O-1$ mRNA was detected in the oral SCC cell lines SAS, HSC3 and HSC4, and biopsy tissue samples from 5 patients with oral SCC. However, the SUMO-1 mRNA was not detected in 3 normal oral epitheliums (Fig. 1B). Therefore, we focused on $S U M O-1$ as a candidate novel gene for a molecular clue to tumorigenesis in oral SCC.

Decrease in proliferation of oral SCC cells transfected with anti-SUMO-1 antisense oligonucleotide. To investigate the role of SUMO-1 on proliferation of oral SCC cells, we transfected anti-SUMO-1 antisense oligonucleotide to SAS cells and analyzed the cell proliferation of the transfectant using MTT assay. SAS cells were transfected $4 \mathrm{~h}$ with anti-SUMO-1 antisense oligonucleotide or control oligonucleotide at the concentration of $0,10,50,100$ and $200 \mathrm{nM}$. Twenty-four hours after transfection, RT-PCR analysis was performed. As shown in Fig. 2A, expression of the SUMO-1 mRNA decreased by transfection with anti-SUMO- 1 antisense oligonucleotide in a dose-dependent manner. In contrast, the expression did not change by transfection with a control oligonucleotide.

The SAS cells transfected with $100 \mathrm{nM}$ anti-SUMO-1 antisense oligonucleotide or $100 \mathrm{nM}$ control oligonucleotide were cultured for $36 \mathrm{~h}$ and their proliferation activities were monitored using MTT assay. As shown in Fig. 2B, proliferation of the anti-SUMO-1 antisense oligonucleotide transfectants was reduced in a culture-time dependent manner. At 24- and 36-h cultures, significant differences were observed between anti-SUMO-1 antisense oligonucleotide transfectants and control oligonucleotide transfectants $(\mathrm{p}<0.01$, each). These results suggested that expression of SUMO-1 is associated with proliferation of oral SCC cells.

SUMO-1 modification of Mdm2 oncoprotein in oral SCC cell lines and tissues. We were interested in oncoprotein Mdm2 as a substrate of SUMO-1 modification associated with cell

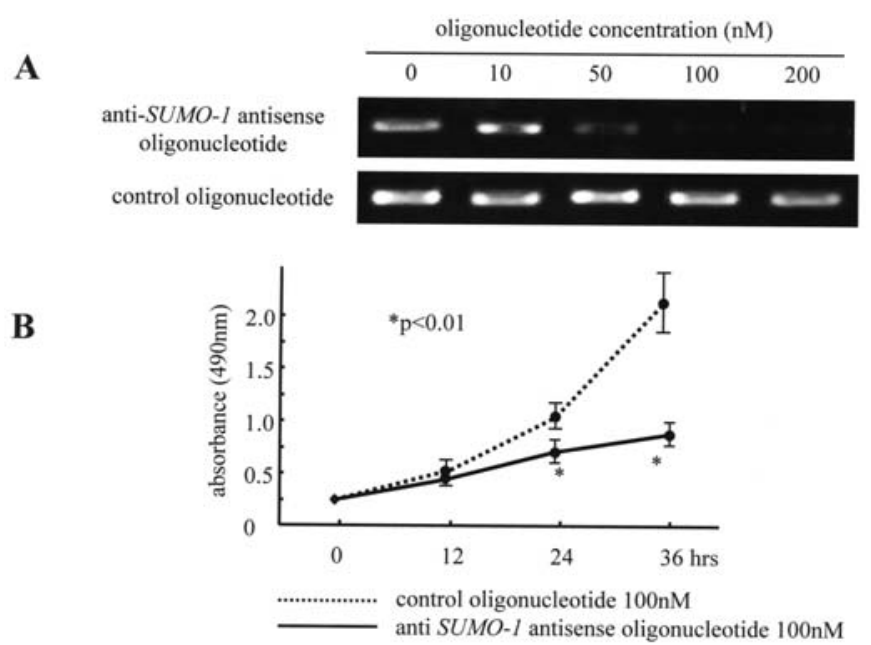

Figure 2. Effects of anti-SUMO-1 antisense oligonucleotide on SUMO-1 expression levels in human oral SCC cell were investigated. (A) SAS cell were transfected with anti-SUMO-1 antisense oligonucleotide or control oligonucleotide at indicated concentration for $4 \mathrm{~h}$. Twenty-four hours after transfection, RT-PCR analysis was performed. A dose-dependent inhabitation of SUMO-1 was observed with 50-200 nM anti-SUMO-1 antisense oligonucleotide. In contrast, control oligonucleotide had no effect on SUMO-1 expression at any concentrations. (B) Effect of anti-SUMO-1 antisense oligonucleotide on the proliferation of SAS was also analyzed. Proliferations of SAS cell transfected with $100 \mathrm{nM}$ anti-SUMO-1 antisense oligonucleotide or $100 \mathrm{nM}$ control oligonucleotide were measured in various time-points using MTT assay. Anti-SUMO-1 antisense oligonucleotide significantly reduced the proliferation SAS cell at 24 and $36 \mathrm{~h}$ after transfection in timedependent manner ( $\mathrm{p}<0.01$ respectively) as compared with control oligonucleotide. The values are the mean \pm SD of five experiments at 490 -nm absorbance. Mann-Whitney U test was used to determine the p-values.

proliferation, because it is reported that Mdm2 sumoylation attenuates its ubiquitination thereby increasing its negative activity towards p53. 


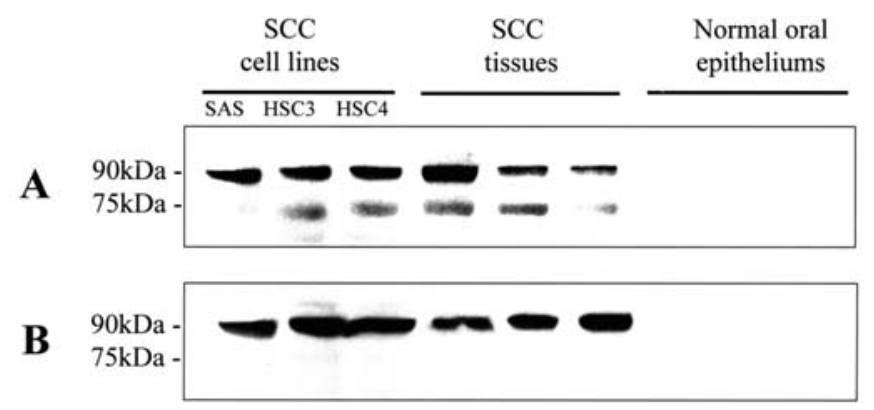

Figure 3. (A) Whole lysates from oral SCC cell lines, SCC tissues and normal oral epitheliums were immunoprecipitated with monoclonal antibody against Mdm2 and analyzed by Western blotting with monoclonal antibody against Mdm2. The $90 \mathrm{kDa}$ and $75 \mathrm{kDa} \mathrm{Mdm} 2$ bands in all oral SCC cells and oral SCC tissues with the monoclonal antibody against Mdm2, although we did not detect these proteins in normal oral epitheliums. The $90 \mathrm{kDa}$ bands were predominantly expressed as compared with $75 \mathrm{kDa}$ bands. (B) Immunoprecipitation with monoclonal antibody against Mdm2 and Western blotting with monoclonal antibody against SUMO-1 detected only the $90 \mathrm{kDa}$ bands.

Mdm2 was immunoprecipitated from lysates of 3 oral SCC cell lines, 3 oral SCC tissues and 3 normal oral epitheliums using anti-Mdm2 monoclonal antibody. Fig. 3 shows Werstern blot profiles of the immunoprecipitated samples using monoclonal antibodies against Mdm2 (Fig. 3A) and SUMO-1 (Fig. 3B). Two Mdm2 bands of 90 and $75 \mathrm{kDa}$ were identified in all oral SCC cells and all oral SCC tissues and the $90 \mathrm{kDa}$ bands were predominantly expressed as compared with $75 \mathrm{kDa}$ bands (Fig. 3A). In Werstern blot analysis with anti-SUMO-1 monoclonal antibody, the $90 \mathrm{kDa}$ bands were stained (Fig. 3B). These findings indicated that the sumoylated $\mathrm{Mdm} 2(90 \mathrm{kDa})$ was present much more than the non-sumoylated Mdm2 (70 kDa) in oral SCC cell lines and oral SCC tissues. On the other hand, any Mdm2 band was not detected in normal oral epitheliums (Fig. 3A).

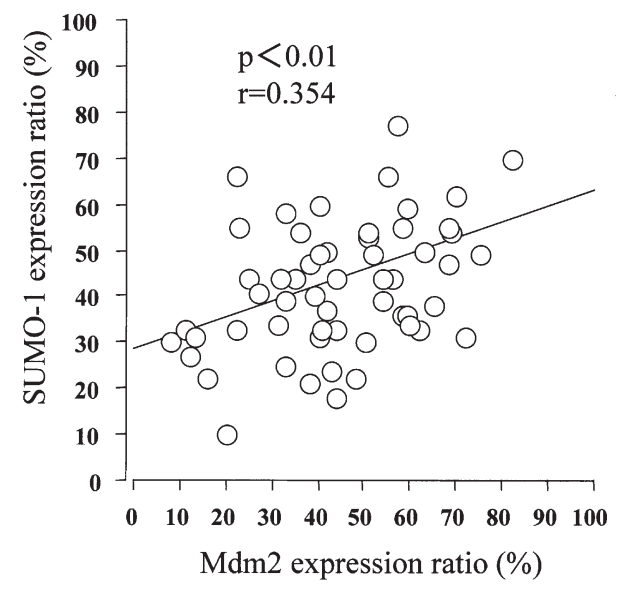

Figure 5. The expression ratio of SUMO-1 positively correlated with that of $\mathrm{Mdm} 2$ in 57 patients with early stage oral SCC $(\mathrm{r}=0.354, \mathrm{p}<0.01)$. Correlation between Mdm2 and SUMO-1 expression ratio was tested by Spearman rank correlation coefficient.

SUMO-1 and Mdm2 expression in oral SCC tissues. We performed immunohistological analysis of SUMO-1 and Mdm2 expressions in biopsy tissue samples from 57 patients with early stage oral SCC and in 5 normal oral epitheliums. The SUMO-1 and Mdm2 expression was hardly detected on normal oral epitheliums. In oral CC tissues, SUMO-1 was expressed on cytoplasm and nucleus of carcinoma cells. Mdm2 was mainly expressed on nucleus of carcinoma cells (Fig. 4). The expression ratio of SUMO-1 in 57 oral SCC tissues was $10-77 \%$ with $44 \%$ of median value. The expression ratio of Mdm2 was $8-82 \%$ with $43 \%$ of median value. There was significant positive correlation between expression ratio of SUMO-1 and Mdm2 (r=0.354, p<0.01; Fig. 5).

Prognostic value of SUMO-1 and Mdm2 expression in early stage of oral SCC. Histological evaluation showed that tumor

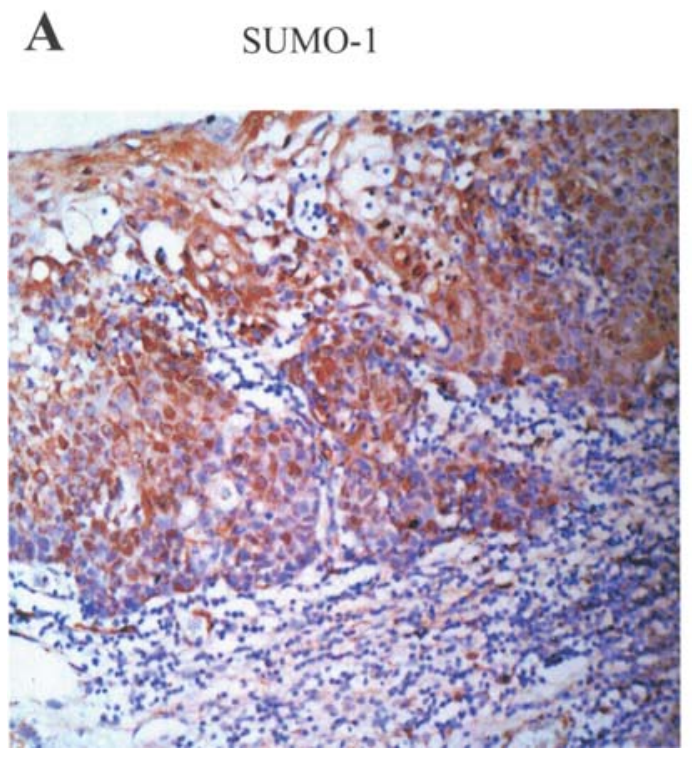

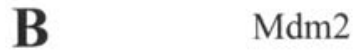

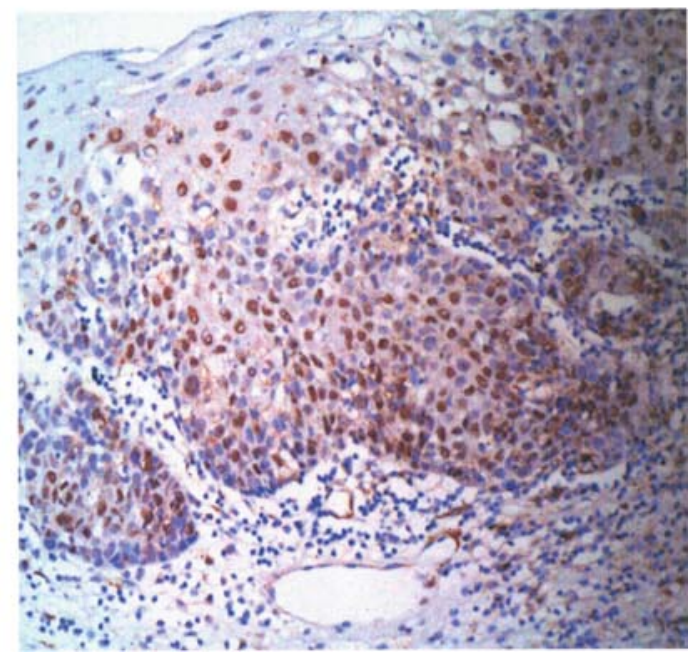

Figure 4. Representative immunohistological features with positive expressions of SUMO-1 and Mdm2 in oral SCC tissues are shown. (A) SUMO-1 was expressed on cytoplasm and nucleus of carcinoma cells. The expressions are cytoplasm dominant. (B) Mdm2 is mainly expressed on nucleus of carcinoma cells (original magnification $\mathrm{x} 200$ ). 

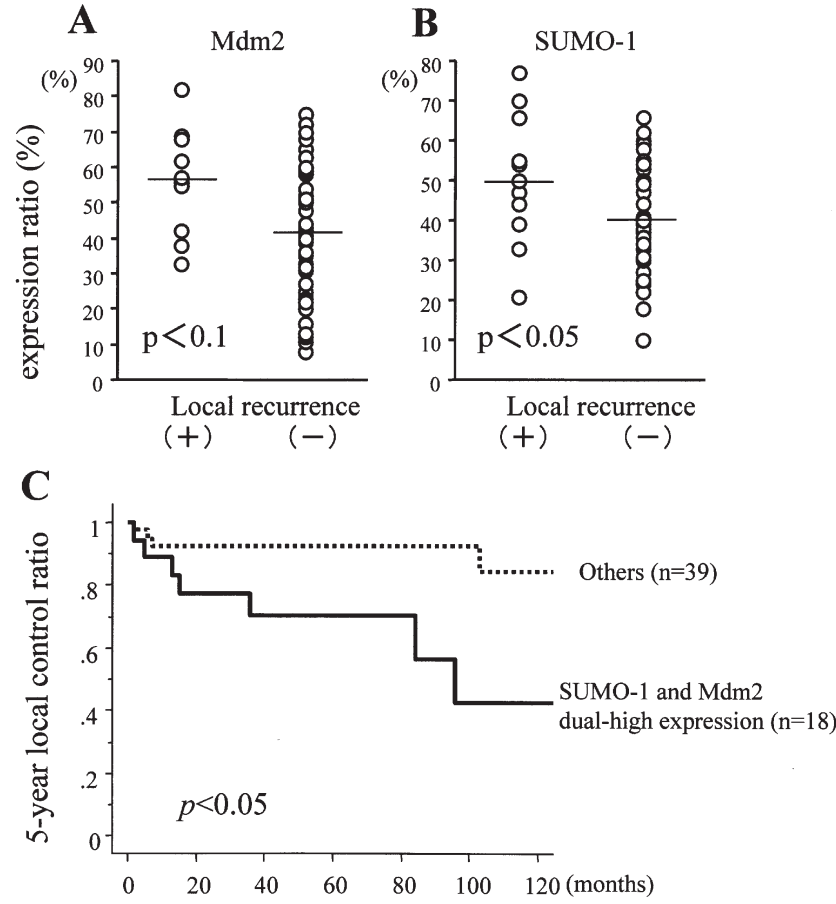

Figure 6. (A) Patients who developed local recurrence showed higher tendency in the Mdm2 expressions ratio than patients without local recurrence $(50.545 \pm 16.489$ vs $.40 .261 \pm 12.632, \mathrm{p}<0.1)$. (B) Patients who developed local recurrence after tumor-free periods showed a significantly higher SUMO-1 expression ratio than patients without local recurrence $(54.545 \pm 15.417$ vs $41.630 \pm 17.495, \mathrm{p}<0.05)$. Results are presented as mean \pm $\mathrm{SD}$. Mann-Whitney U test was used to determine the p-value. (C) The 5year local control rates of patients with the dual-high expression of SUMO-1 and $\mathrm{Mdm} 2$ were significantly shorter than that of the other patients $(\mathrm{p}<0.05)$. The probabilities of local control were calculated using the Kaplan-Meier method and compared using the log-rank test.

cells were not present on the surgical margin from any of the 51 patients that underwent surgery. On this evaluation, there were no tumor cells in the biopsy samples obtained from any of the 6 patients after radiotherapy alone. Therefore, all patients experienced a disease-free period.

However, 11 (19\%) of 57 patients developed local recurrence. Of 11 patients with local recurrence, six had successful salvage surgery, four (37\%) developed also regional lymph node metastasis and three $(27 \%)$ had also both regional lymph node and distant metastases. Of 46 patients without local recurrence, four (9\%) developed regional lymph node metastasis and six (13\%) had both regional lymph node and distant metastases. Eighteen (32\%) patients died during follow-up; 12 (21\%) of them died of tumor-related disorder and $6(11 \%)$ were tumor-free and died from intercurrent diseases. The 5-year LC, RDC, OS and CSS, were: $85.1 \%, 80.8,72.7$ and $82.9 \%$, respectively.

Gender, age, histology and T-classification at diagnosis did not influence local recurrence, regional lymph node and distant metastases. Patients who developed local recurrence tended to have a higher Mdm2 expression ratio than patients without local recurrence $(50.545 \pm 16.489$ vs. $40.261 \pm 12.632$, $\mathrm{p}<0.1$; Fig. 6A). Patients who developed local recurrence after tumor-free periods showed a significantly higher SUMO-1 expression ratio than patients without local recurrence $(54.545 \pm 15.417$ vs $41.630 \pm 17.495, \mathrm{p}<0.05$; Fig. 6B).
Table II. A, Univariate Cox proportional hazards analysis for local control of variables in 57 patients with early stage oral squamous cell carcinoma.

\begin{tabular}{lccc}
\hline Variable & $\begin{array}{c}\text { Hazard } \\
\text { ratio }\end{array}$ & 95\% CI & p-value \\
\hline Age $(\geq 60)$ & 1.149 & $0.330-4.001$ & 0.8268 \\
Gender (male) & 1.339 & $0.160-3.490$ & 0.7107 \\
Primary site (floor of mouth) & 2.587 & $0.728-9.149$ & 0.1417 \\
Tumor differentiation & 0.842 & $0.182-3.902$ & 0.8262 \\
(poorly/moderately) & & & \\
Clinical stage (II) & 3.230 & $0.943-11.066$ & 0.0620 \\
SUMO-1 and Mdm2 dual- & 4.386 & $0.066-0.784$ & 0.0190 \\
high expression & & & \\
\hline
\end{tabular}

B, Multivariate Cox proportional hazards analysis for local control of variables in 57 patients with early stage oral squamous cell carcinoma.

\begin{tabular}{lccc}
\hline Variable & $\begin{array}{c}\text { Hazard } \\
\text { ratio }\end{array}$ & $95 \%$ CI & p-value \\
\hline Age $(\geq 60)$ & 0.992 & $0.240-4.107$ & 0.9914 \\
Gender (male) & 1.158 & $0.196-6.813$ & 0.8728 \\
Primary site (floor of mouth) & 1.907 & $0.462-7.870$ & 0.3723 \\
Tumor differentiation & 0.892 & $0.159-4.994$ & 0.8969 \\
(poorly/moderately) & & & \\
Clinical stage (II) & 3.955 & $1.100-14.225$ & 0.0352 \\
SUMO-1 and Mdm2 dual- & 5.155 & $0.050-0.757$ & 0.0182 \\
high expression & & & \\
\hline
\end{tabular}

CI, confidence interval.

However, the expression ratio of SUMO-1 and Mdm2 were not different between patients with or without regional lymph node metastases or distant metastasis.

According to expression ratio of SUMO-1 and Mdm2 respectively, we categorized 57 patients into 2 groups by the median value of $43 \%$ each. Twenty-nine patients were in the high SUMO-1 expression and 28 were in the high Mdm2 expression group. There was no significant difference between patients with high SUMO-1 expression and patients with low SUMO-1 expression in OS, CSS, LC and RDC with high Mdm2 expression and patients with low Mdm2 expression in OS, CSS, LC and RDC. However, the 5-year LC rates of patients with dual-high expression of SUMO-1 and $\mathrm{Mdm} 2$ were significantly shorter than that of the other patients $(\mathrm{p}<0.05$; Fig. 6C). Univariate analysis also showed that dual-high expression of SUMO-1 and Mdm2 was a significant indicator for poor local control (hazard ratio: 4.386, confidence interval: 0.066-0.784, $\mathrm{p}=0.0190$; Table IIA). Multivariate analysis confirmed that dual-high expression of SUMO-1 and Mdm2 was an independent factor 
for local failure (hazard ratio 5.155, confidence interval 0.050-0.757, $\mathrm{p}=0.0182$; Table IIB).

\section{Discussion}

In this study, we preliminarily performed cDNA array analysis to study the difference of gene expression profiles among normal oral epithelium, oral SCC tissues and oral SCC cell lines. Among several candidate genes that expressed at higher levels in oral SCC, we focused on SUMO-1 as a novel gene for a molecular clue to tumor genesis in oral SCC. SUMO-1 is a ubiquitin-like protein and is reported to be conjugated, like ubiquitin, by a set of enzymes to cellular regulatory proteins, including oncogenes and tumor suppressor genes $(4-6,20)$. Given the substrates involved, protein sumoylation would be expected to be important in the course of tumorigenesis and, accordingly, altered in human cancer (7). However, evidence to support this notion is scarce. In this study, RT-PCR analysis as well as cDNA array analysis showed that the SUMO-1 gene expressed in human oral SCC tissues and cell lines at much higher levels than in normal oral epithelium. Immunohistological analysis revealed also that the SUMO-1 expression was detected in oral SCC tissues, but was hardly detected in normal oral epitheliums. Transfection of the anti-SUMO-1 antisense oligonucleotide to oral SCC cells significantly reduced proliferation of the cells, suggesting that SUMO-1 may be involved in tumor proliferation. This is the first report that SUMO- 1 is expressed in human oral SCC in vitro and in vivo at much higher level than in normal oral epithelium and may have a role in tumor cell proliferation.

A relatively small number of oncoproteins such as PML (promyelocytic leukemia), c-Myb and Mdm2, have been found to undergo sumoylation; it is expected that the list will grow as studies proceed and reagents that can inhibit efficient desumoylation are developed. Among these oncogenes, we were interested in oncoprotein Mdm2 as a substrate of SUMO-1 modification associated with cell proliferation. Mdm2 is a RING finger protein with E3 ubiquitin ligase activity whose oncogenic potential has been attributed in part to its ability to target the p53 tumor suppressor protein for ubiquitination and degradation (10-12).

In this study, using immunoprecipitation and Western blot analyses, we showed clearly that the Mdm2 presented predominantly as a sumoylated isoform rather than as a nonsumoylated isoform in oral SCC tissues and oral SCC cell lines. Significant positive correlation between SUMO-1 and Mdm2 expressions found in 57 patients with oral SCC indicates that $\mathrm{Mdm} 2$ may be sumoylated in vivo at the constant ratio regardless of $\mathrm{Mdm} 2$ expression levels. Desumoylation of $\mathrm{Mdm} 2$ is reported to promote selfubiquitination and degradation of $\mathrm{Mdm} 2$, resulting in prevention of p53 degradation (13). Such alternation of p53 is well known to inhibit p53-mediated transactivation of genes related to cell cycle arrest and apoptosis $(21,22)$. Therefore, it is possible that sumoylated Mdm2 may increase ubiquitination and degradation of p53, resulting in tumor cell proliferation in oral SCC, as shown previously in in vitro studies. Alternatively, another sumoylated cell cycle regulatory proteins that have not been reported yet may be involved. Further studies are expected to reveal whether corresponding cell cycle regulatory proteins are also sumoylated and the possible effects of alterations in such processes in the course of tumorigenesis.

We also investigated whether expression of SUMO-1 and/or Mdm2 correlates with patient prognoses in 57 patients with early stage (I-II) oral SCC. Our patients were treated in 3 different manners. The metastasis in regional lymph nodes and/or distant metastasis or patient survival was not influenced by the treatment modality. Furthermore, there was no difference in expressions of SUMO-1 or Mdm2 among the 3 treatment groups. Therefore, analyses for correlation between SUMO-1 or Mdm2 expression and the metastasis or patient prognosis were not influenced by treatment modality. In our series of early stage oral SCC, the expressions of SUMO-1 and Mdm2 did not influence RDC, OS and CSS. However, patients who developed local recurrence after treatments tended to have a higher Mdm2 expression ratio than the patients without local recurrence, moreover, such patients showed a significantly higher SUMO-1 expression ratio than patients without local recurrence. With regards to clinical outcomes, patients with dual-high expression of SUMO-1 and Mdm2 had poorer local control than the other patients.

Multivariate analysis revealed that dual-high expression of SUMO-1 and Mdm2 was an independent factor for local failure. This is the first report regarding prognostic value of dual-high expression of SUMO-1 and Mdm2 in human cancer. Although there is certain number of reports regarding expression of Mdm2 in human cancer, its prognostic value is still controversial. The Mdm2 overexpression was reported to be associated with poor prognosis in breast cancer $(14,15)$, esophageal carcinoma (16), hepatocellular carcinoma (17) and renal cell carcinoma (18). However, no association between $\mathrm{Mdm} 2$ expression and patient prognosis was reported in breast cancer (23), colorectal carcinoma (24), bladder cancer (25), or prostate cancer (26). On the contrary, the expression was reported to be associated with improved survival in malignant melanoma $(21,27)$. The prognostic value of the Mdm2 expression may vary among tumor type and/or stage. Further studies will be necessary to define the prognostic value of SUMO-1 expression in human cancer.

In conclusion, we showed that $S U M O-1$ is overexpressed in human oral SCC in vitro and in vivo and may be involved in tumor cell proliferation. Using immunoprecipitation and Western blot analyses, we showed clearly that the Mdm2 presented predominantly as a sumoylated isoform in oral SCC tissues and oral SCC cell lines. With regards to clinical outcomes, dual-high expression of SUMO-1 and Mdm2 was associated with local recurrence in early stage oral SCC. These results suggest that overexpression of $\mathrm{Mdm} 2$ caused by overexpression of SUMO-1 may be involved in tumor aggressiveness even in patients with early stages of oral SCC. As anti-tumor effect of anti-Mdm2 antisense oligonucleotide recently has been reported by several investigators (28-30), our result suggest that SUMO-1 will be useful not only as a clinical indicator for local control together with Mdm2, but may also be a novel target for therapy in patients with oral SCC. 


\section{Acknowledgements}

This work was supported by Grant-in-Aid for scientific research from the Ministry of Education, Science and Culture, Japan.

\section{References}

1. Hochstrasser M: Evolution and function of ubiquitin-like proteinconjugation systems. Nat Cell Biol 2: 153-157, 2000.

2. Yeh ET, Gong L and Kamitani T: Ubiquitin-like proteins: new wines in new bottles. Gene 248: 1-14, 2000.

3. Melchior F: SUMO - nonclassical ubiquitin. Annu Rev Cell Dev Biol 16: 591-626, 2000.

4. Sternsdorf T, Jensen K and Will H: Evidence for covalent modification of the nuclear dot-associated proteins PML and Sp100 by PIC1/SUMO-1. J Cell Biol 139: 1621-1634, 1997.

5. Bies J, Markus J and Wolff L: Covalent attachment of the SUMO-1 protein to the negative regulatory domain of the c-Myb transcription factor modifies its stability and transactivation capacity. J Biol Chem 277: 8999-9009, 2002.

6. Muller S, Berger M, Lehembre F, Seeler JS, Haupt Y and Dejean A: c-Jun and p53 activity is modulated by SUMO-1 modification. J Biol Chem 275: 13321-13329, 2000.

7. Alarcon-Vargas D and Ronai Z: SUMO in cancer - wrestlers wanted. Cancer Biol Ther 1: 237-242, 2002.

8. Xirodimas DP, Chisholm J, Desterro JM, Lane DP and Hay RT: P14ARF promotes accumulation of SUMO-1 conjugated (H)Mdm2. FEBS Lett 528: 207-211, 2002.

9. Chen L and Chen J: MDM2-ARF complex regulates p53 sumoylation. Oncogene 22: 5348-5357, 2003.

10. Haupt Y, Maya R, Kazaz A and Oren M: Mdm2 promotes the rapid degradation of p53. Nature 387: 296-299, 1997.

11. Kubbutat MH, Jones SN and Vousden KH: Regulation of p53 stability by Mdm2. Nature 387: 299-303, 1997.

12. Li M, Brooks CL, Wu-Baer F, Chen D, Baer R and Gu W: Mono- versus polyubiquitination: differential control of $\mathrm{p} 53$ fate by Mdm2. Science 302: 1972-1975, 2003.

13. Lee MH, Lee SW, Lee EJ, Choi SJ, Chung SS, Lee JI, Cho JM, Seol JH, Baek SH, Kim KI, Chiba T, Tanaka K, Bang OS and Chung CH: SUMO-specific protease SUSP4 positively regulates p53 by promoting Mdm2 self-ubiquitination. Nat Cell Biol 8: 1424-1431, 2006.

14. Cuny M, Kramar A, Courjal F, Johannsdottir V, Iacopetta B, Fontaine H, Grenier J, Culine S and Theillet C: Relating genotype and phenotype in breast cancer: an analysis of the prognostic significance of amplification at eight different genes or loci and of p53 mutations. Cancer Res 60: 1077-1083, 2000.

15. Jiang M, Shao ZM, Wu J, Lu JS, Yu LM, Yuan JD, Han QX, Shen ZZ and Fontana JA: p21/waf1/cip1 and mdm-2 expression in breast carcinoma patients as related to prognosis. Int J Cancer 74: 529-534, 1997.

16. Shimada Y, Imamura M, Shibagaki I, Tanaka H, Miyahara T, Kato $\mathrm{M}$ and Ishizaki K: Genetic alterations in patients with esophageal cancer with short- and long-term survival rates after curative esophagectomy. Ann Surg 226: 162-168, 1997.
17. Endo K, Ueda T, Ohta T and Terada T: Protein expression of MDM2 and its clinicopathological relationships in human hepatocellular carcinoma. Liver 20: 209-215, 2000.

18. Uchida T, Gao JP, Wang C, Jiang SX, Muramoto M, Satoh T, Minei S, Shimura S, Irie A, Kameya T and Baba S: Clinical significance of $\mathrm{p} 53, \mathrm{mdm} 2$, and bcl-2 proteins in renal cell carcinoma. Urology 59: 615-620, 2002.

19. Bandoh N, Hayashi T, Kishibe K, Takahara M, Imada M, Nonaka S and Harabuchi Y: Prognostic value of p53 mutations, bax, and spontaneous apoptosis in maxillary sinus squamous cell carcinoma. Cancer 94: 1968-1680, 2002.

20. Duprez E, Saurin AJ, Desterro JM, Lallemand-Breitenbach V, Howe K, Boddy MN, Solomon E, De The H, Hay RT and Freemont PS: SUMO-1 modification of the acute promyelocytic leukaemia protein PML: implications for nuclear localisation. J Cell Sci 112: 381-393, 1999.

21. Momand J, Zambetti GP, Olson DC, George D and Levine AJ: The $\mathrm{mdm}-2$ oncogene product forms a complex with the p53 protein and inhibits p53-mediated transactivation. Cell 69: 1237-1245, 1992.

22. Momand J and Zambetti GP: Mdm-2: 'big brother' of p53. J Cell Biochem 64: 343-352, 1997.

23. Marchetti A, Buttitta F, Girlando S, Dalla Palma P, Pellegrini S, Fina P, Doglioni C, Bevilacqua G and Barbareschi M: mdm2 gene alterations and $\mathrm{mdm} 2$ protein expression in breast carcinomas. $\mathrm{J}$ Pathol 175: 31-38, 1995.

24. Abdel-Fattah G, Yoffe B, Krishnan B, Khaoustov V and Itani K: MDM2/p53 protein expression in the development of colorectal adenocarcinoma. J Gastrointest Surg 4: 109-114, 2000.

25. Pfister C, Moore L, Allard P, Larue H, Lacombe L, Tetu B, Meyer $\mathrm{F}$ and Fradet $\mathrm{Y}$ : Predictive value of cell cycle markers p53, MDM2, p21, and Ki-67 in superficial bladder tumor recurrence. Clin Cancer Res 5: 4079-4084, 1999.

26. Osman I, Drobnjak M, Fazzari M, Ferrara J, Scher HI and Cordon-Cardo C: Inactivation of the p53 pathway in prostate cancer: impact on tumor progression. Clin Cancer Res 5: 2082-2088, 1999.

27. Polsky D, Melzer K, Hazan C, Panageas KS, Busam K, Drobnjak M, Kamino H, Spira JG, Kopf AW, Houghton A, Cordon-Cardo C and Osman I: HDM2 protein overexpression and prognosis in primary malignant melanoma. J Natl Cancer Inst 94: 1803-1806, 2002.

28. Wang H, Zeng X, Oliver P, Le LP, Chen J, Chen L, Zhou W, Agrawal S and Zhang R: MDM2 oncogene as a target for cancer therapy: an antisense approach. Int J Oncol 15: 653-660, 1999.

29. Chen L, Agrawal S, Zhou W, Zhang R and Chen J: Synergistic activation of $\mathrm{p} 53$ by inhibition of MDM2 expression and DNA damage. Proc Natl Acad Sci USA 95: 195-200, 1998.

30. Chen L, Lu W, Agrawal S, Zhou W, Zhang R and Chen J: Ubiquitous induction of p53 in tumor cells by antisense inhibition of MDM2 expression. Mol Med 5: 21-34, 1999. 